\title{
Criticality in amorphous topological matter: Beyond the universal scaling paradigm
}

\author{
Moein N. Ivaki॰, Sahlberg Isac, and Teemu Ojanen $\odot$ \\ Computational Physics Laboratory, Physics Unit, Faculty of Engineering and Natural Sciences, Tampere University, \\ P.O. Box 692, FI-33014 Tampere, Finland and Helsinki Institute of Physics P.O. Box 64, FI-00014, Finland
}

(Received 11 June 2020; accepted 2 November 2020; published 1 December 2020)

\begin{abstract}
We establish the theory of critical transport in amorphous Chern insulators and show that it lies beyond the current paradigm of topological criticality epitomized by the quantum Hall transitions. We consider models of Chern insulators on percolation-type random lattices where the average density determines the statistical properties of geometry. While these systems display a two-parameter scaling behavior near the critical density, the critical exponents and the critical conductance distributions are strikingly nonuniversal. Our analysis indicates that the amorphous topological criticality results from an interpolation of a geometric-type transition at low density and an Anderson localization-type transition at high density. Our work demonstrates how the recently discovered amorphous topological systems display unique phenomena distinct from their conventionally studied counterparts.
\end{abstract}

DOI: 10.1103/PhysRevResearch.2.043301

\section{INTRODUCTION}

Recent theoretical advances have brought the full topological classification of crystalline matter in sight [1-3]. However, there are rapidly emerging lines of research in topological systems without spatial symmetry. Since nontrivial topology in general does not rely on spatial order, amorphous systems provide an interesting new platform for topological matter [4-21]. Previously, the question as to whether the topological behavior of amorphous systems and crystalline systems display fundamental differences has remained largely unclear. In this work we answer this question affirmatively by establishing that the critical transport of amorphous Chern insulators exhibit striking departures from their spatially ordered counterparts.

The theory of quantum Hall $(\mathrm{QH})$ plateau transitions, initiated by Khmelnitskii and Pruisken [22,23], has achieved a paradigmatic role in the theory of topological phase transitions. This theory, with generalizations to various symmetry classes and models, describes topological phase transitions as a form of Anderson localization (AL) transition with diverging localization length (LL) [24,25]. The topological phase transition corresponds to an unstable fixed point, characterized by universal critical exponents, in a two-parameter space. This picture, with appropriate modifications, is believed to capture the generic features of topological phase transition in noninteracting systems. In particular, the transitions are classified by a set of universal critical exponents that only depend on the symmetries and generic features of the system

Published by the American Physical Society under the terms of the Creative Commons Attribution 4.0 International license. Further distribution of this work must maintain attribution to the author(s) and the published article's title, journal citation, and DOI. but not on microscopic details. While theoretically predicted values for the LL exponents in the $\mathrm{QH}$ transition exhibit a degree of variation and seem to somewhat overestimate the experimental ones [26-31], the values extracted from widely different models typically fall in the range $v=2.4-2.6$ [32-43]. This degree of agreement lends significant credibility to the orthodox theory. In the present work we establish the critical theory of amorphous Chern insulators and show that it lies strikingly beyond the universal scaling paradigm. We study transport properties of amorphous topological states defined on random lattices with variable density as depicted in Fig. 1(a). By numerically evaluating configuration-averaged longitudinal and Hall conductivities $\sigma_{x x}, \sigma_{x y}$ in setups illustrated in Figs. 1(b) and 1(c), we study their scaling behavior as a function of density. While conductivities are shown to obey two-parameter scaling behavior near the critical density $\rho_{c}$, the critical exponent $v$ characterizing the diverging $\mathrm{LL}$ as $\xi \propto$ $\left|\rho-\rho_{c}\right|^{-v}$ is strongly nonuniversal $v=1.01(1)-1.35(2)$. To further characterize the nonuniversality, we calculate the critical conductance distributions (CDs) and show how they interpolate between two distinct types, one which exhibits QH-type features at high density, and another which exhibits a striking low-conductance peak stemming from geometric fluctuations at low density. We conclude that the amorphous topological criticality (ATC) arises from the interpolation of a geometric percolation-type and the AL-type transitions.

\section{MODELS OF AMORPHOUS CHERN INSULATORS}

Following Ref. [44], we study two-band Chern insulators with the tight-binding Hamiltonian

$$
H=\left(\begin{array}{cc}
(2-M) \delta_{i j}+T_{i j} & i T_{i j} e^{-i \phi_{i j}} \\
i T_{i j} e^{i \phi_{i j}} & -(2-M) \delta_{i j}-T_{i j}
\end{array}\right)
$$


(a)

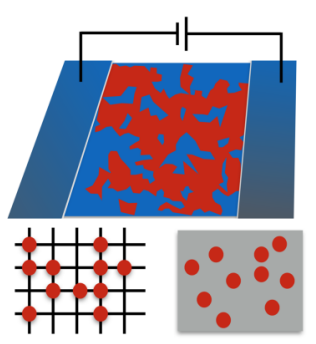

(b)

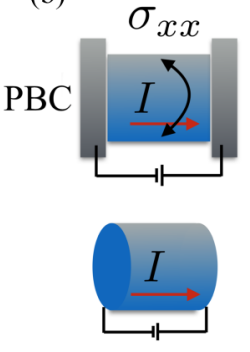

(c)

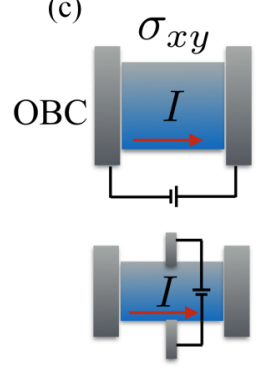

FIG. 1. (a) Schematic setup for transport studies in amorphous Chern insulators. The studied random geometries are generated by discrete and continuum percolation lattices. (b) Longitudinal conductivity can be extracted from the two-terminal conductance with periodic boundary conditions in the transverse direction (top) which is equivalent to the setup below. (c) Hall conductivity can be extracted from the two-terminal setup with open boundary conditions (top). The conductivity corresponds to the Hall conductivity obtained from the four-terminal setup (bottom).

where $M$ is the time-reversal breaking mass term in the units of a characteristic hopping amplitude and $T_{i j}=$ $-\frac{1}{2} e^{-r_{i j} / \eta} \theta\left(R-r_{i j}\right)$ describes the spatial decay of the hopping amplitudes. Here $r_{i j}=\left|\mathbf{r}_{i}-\mathbf{r}_{j}\right|$ is the distance between sites $i, j$, the parameters $\eta, R$ describe the decay of hopping, and the phase factor is given by $e^{i \phi_{i j}}=\left(r_{i j}^{x}+i r_{i j}^{y}\right) / r_{i j}$, where $r_{i j}^{x}=x_{i}-x_{j}$. We mainly consider disk hopping models with $\eta=\infty$ but also check that the discovered qualitative features are present for smooth spatial decay with constant $\eta$ and
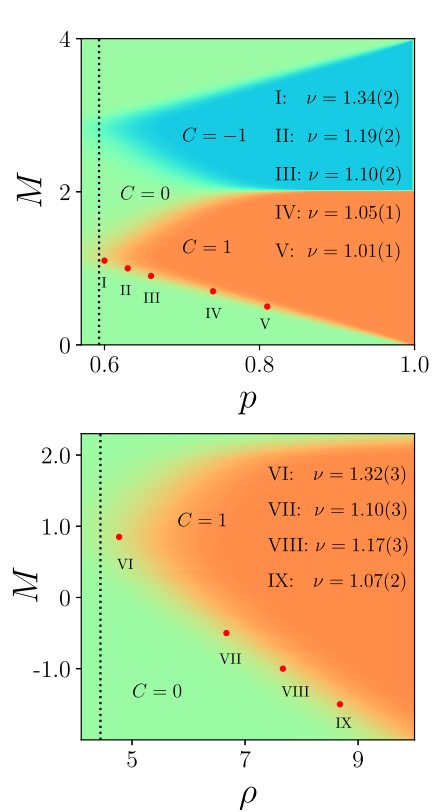

(b)
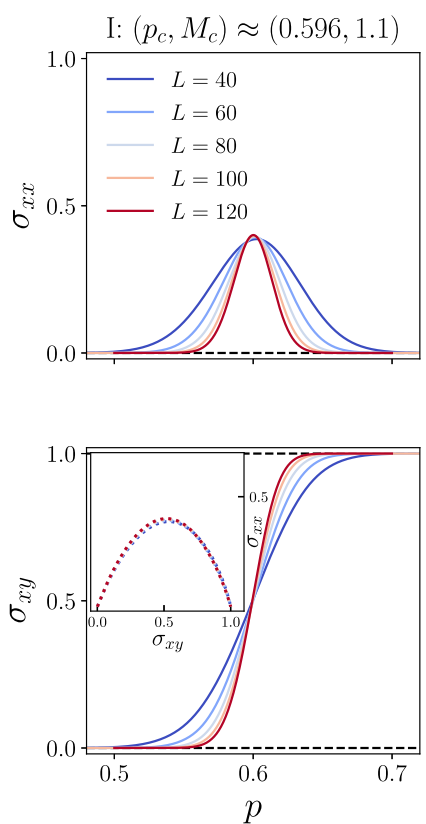

$R \rightarrow \infty$. The studied model belongs to the Altland-Zirnbauer symmetry class $\mathrm{D}$.

We study the model (1) on random percolation-type geometries on a square lattice as well as in a continuum, as illustrated in Fig. 1(a). As in percolation theory, the lattice sites in the discrete case are independently populated with probability $p$, whereas in the continuum problems the sites are independently distributed in the two-dimensional (2d) continuum with intensity $\rho$ particles per unit area.

\section{SCALING THEORY OF TRANSPORT}

We assume that the electronic states are half filled (one electron per site) and study electrical conductance averaged over different random configurations as a function of the density of lattice sites. More precisely, in the discrete case we study the topological criticality as a function of $p$ and in the continuum case as a function of $\rho$. For discrete random realizations, we evaluate conductances by employing the KWANT package [45]. For continuum configurations, we employ the Green's function method outlined in Sec. I of the Supplemental Materials (SM) [46]. The longitudinal and Hall conductivities are obtained from square-shaped samples in the two-terminal setups illustrated in Figs. 2(b) and 2(c). The correspondence between the Hall conductivity obtained from the four-terminal setup and the two-terminal setup is illustrated in Sec. III in the SM [46-48]. The central piece of computational technology in our work is to carry out the configuration averages with fixed number of lattice sites $n$ and subsequently exploit the analytical connection between $n$ and $p(\rho)$. In Sec. I of the SM [46] we show that this procedure

(c)
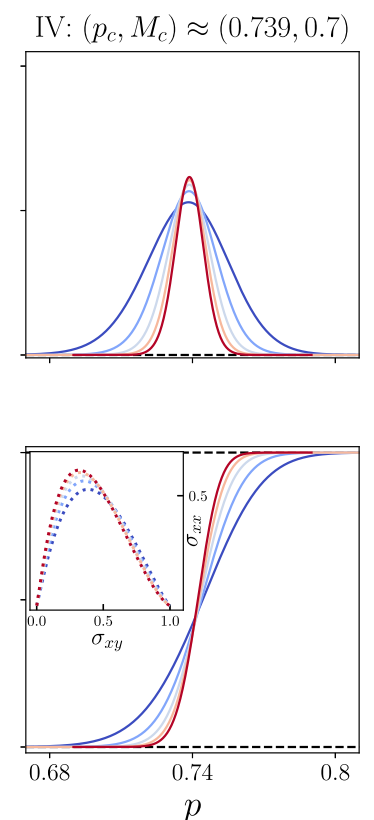

(d)
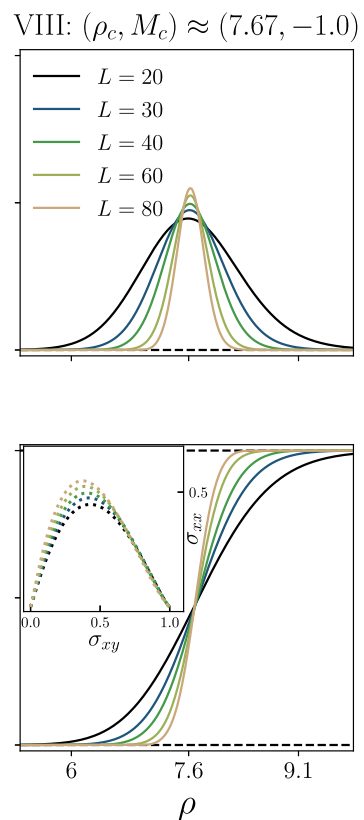

FIG. 2. (a) Topological phase diagram (Chern numbers) in the density-mass plane for the discrete (top) and the continuum (bottom) model. The red dots labeled by roman numerals indicate the positions where the scaling analysis was carried out. The black dotted line indicates the percolation threshold of the lattice. (b) Conductance scaling in the lattice model at the optimal point I. The inset in the bottom shows the flow in the conductivity plane. (c) Same as panel (b) but in a higher-density regime. (d) Conductance scaling in the continuum model at VIII. The curves are generated from over $10^{5}$ configurations. 
significantly reduces the statistical fluctuations compared with direct sampling of $p$.

We postulate that the conductivities for density-driven topological transition satisfy a two-parameter scaling form

$$
\sigma=F\left[L^{1 / v} \zeta_{1}(p), L^{y} \zeta_{2}(p)\right],
$$

where $L$ is the linear system size, $v$ is the critical exponent of localization length, and $y<0$ describes the irrelevant scaling direction [49]. Here $F(x, y)$ is an a priori unknown scaling function and $\zeta_{1}(p), \zeta_{2}(p)$ describe the relevant and irrelevant scaling variables, respectively. In the large-system limit we recover a single-parameter scaling characterized by the LL $\xi \propto\left|p-p_{c}\right|^{-v}$ where $p_{c}$ is the critical density. For continuum problems we postulate a similar expression with $p$ and $p_{c}$ substituted by particle intensity per unit area $\rho$ and its critical value $\rho_{c}$. The statistical analysis [50] of extracting $v, p_{c}$, and $y$ from the conductance data is presented in Sec. II in the SM [46].

The topological phase diagrams of lattice and continuum disk hopping models are evaluated following Ref. [44] and illustrated in Fig. 2(a). The red dots indicate the points I-V and VI-IX on the phase boundary where the critical parameters have been evaluated. The localization exponents are listed in Fig. 2(a) and the full scaling data are presented in Tables I to III in the SM [46]. The behavior of conductivities as a function of density is illustrated in Figs. 2(b)-2(d). In general, we obtain an excellent fit of the conductance data with the two-parameter scaling form at each studied point. For discrete and continuum disk models we observe that the nontrivial phase reaches down to the percolation threshold which is the theoretical lower limit for the topological phase for these models [44]. The critical density $p_{c}=0.596(2)$ at point I matches well the percolation threshold of square lattice $p_{c}^{c l} \approx 0.593$. Also, the LL exponents at peak points I $[v=1.34(2)]$ and VI $[v=1.32(3)]$ are in excellent agreement with the correlation length exponent $4 / 3$ of $2 \mathrm{~d}$ percolation [51]. These results together indicate that when the critical density approaches the geometric percolation threshold of the lattice, the critical wave functions are restricted only by the geometry of the underlying lattice, not quantum interference effects.

At higher densities away from I and VI, the critical exponents do not agree with the low-density value and show large nonuniversal variation. This remarkable behavior is in in striking contrast with the universal behavior of the class D disordered systems for which the exponent has the wellknown value $v=1$ [25]. This value is reached in the studied system only in $p \rightarrow 1$ regime. We observe continuous variation of critical exponents $v=1.01(1)-1.35(2)$ for the discrete model and similar for the continuum disk model. As listed in the SM [46], the critical conductance values also exhibit large nonuniversal variation. This is in sharp contrast with QH systems, where the universality of $\sigma_{x x}^{c}$ [52-55] is believed to follow from universal multifractal properties [40,56-58]. The strong variation of the critical properties suggests that topological phase transitions at high- and low-density regimes are dominated by qualitatively different mechanisms. At low density, the agreement of $v$ and $p_{c}$ with the correlation length exponent and the threshold in classical percolation suggest that the reduced lattice connectivity drives the transition. The conductance distribution functions calculated below confirm this observation as well as suggest that the transition at high densities is dominated by conventional AL mechanism.

We note that the topological transition can also be induced at fixed density by varying the mass parameter $M$ through a critical point $\left(p_{c}, M_{c}\right)$ on a phase boundary. Since conductance is an analytic function of $p$ and $M$ for finite systems, the exponent $v^{\prime}$ characterizing the divergence $\xi_{M} \propto\left|M-M_{c}\right|^{-v^{\prime}}$ is expected to coincide with the critical exponent $v$ in the density-driven transition. Indeed, in Sec. V in the SM [46] we illustrate that the two exponents are consistent.

\section{CRITICAL CONDUCTANCE DISTRIBUTIONS}

To gain better insights into the critical behavior, we now study the critical conductance distribution functions (see Sec. III in the SM [46] for technical details). Figures 3(a)-3(c) illustrate the behavior of the longitudinal CDs at I, II, IV (top row) and VI, VII, IX (bottom row) indicated in Fig. 2. At high densities, distributions are qualitatively similar to the one shown in Fig. 3(a), illustrating that the conductance is broadly distributed between 0 and 1 (in the units of $e^{2} / h$ ) with a tendency to peak when approaching 1 . The variance of conductance is clearly scale invariant at $p_{c}$ (up to weak finite-size corrections) and exhibits a double-peak feature reminiscent of the one observed in the $\mathrm{QH}$ transition [59]. These properties are qualitatively similar to those of critical distributions in disordered systems [56,60-66].

When decreasing density towards the threshold I (or VI), the $\mathrm{CD}$ acquires a peak near zero conductance [Fig. 3(b)], ultimately becoming a delta peak when density approaches the percolation threshold of the lattice [Fig. 3(c)]. At the threshold, the CD can be expressed as $f_{p_{c}}(\sigma)=(1-\alpha) \delta(\sigma)+$ $\alpha h(\sigma)$ with $0<\alpha<1$ denoting the fraction of connected lattice configurations. Here $h$ is a normalized distribution which controls the finite conductance part. The striking appearance of the low-conductance peak is a consequence of the vicinity of the percolation threshold, where $50 \%$ of the configurations become disconnected with vanishing conductance. In the thermodynamic limit, the zero-conductance $\delta$ function will vanish above the percolation threshold but unavoidably leaves behind a nonsingular low-conductance peak. Interestingly, the double-peak feature of the variance near $p_{c}$ is not observed at low densities.

The CDs for $\sigma_{x y}$ are shown in Fig. 3(d). At high densities, the distributions of $\sigma_{x y}$ and $\sigma_{x x}$ show strong qualitative differences as in the $\mathrm{QH}$ systems [65]. However, when approaching the threshold I (or VI), both distributions acquire a similar form. This further reinforces the fact that the critical behavior at low and high densities is dominated by distinct mechanisms. Since the distribution functions in discrete and continuum geometries (including the exponential hopping model studied in Sec. VI in the SM [46]) lead to qualitatively similar conclusions, we identify the low-conductance peak as a generic characteristic of ATC.

Together, the conductance scaling and the CDs provide a compelling evidence that the remarkable characteristics of ATC arise from the interpolation of a geometric-type transition at low and conventional localization-type transition at high densities. Near the threshold I (or VI), the localization length exponent and the $\mathrm{CD}$ functions are consistent with the 
(a)

IV
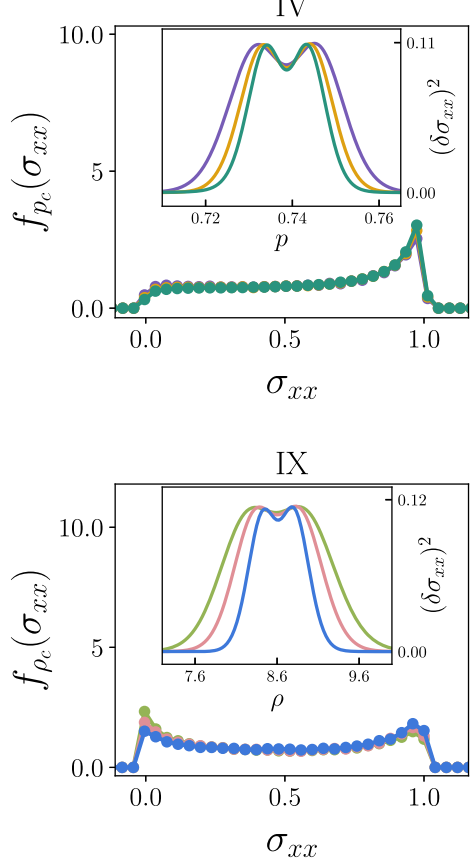

(b)

II

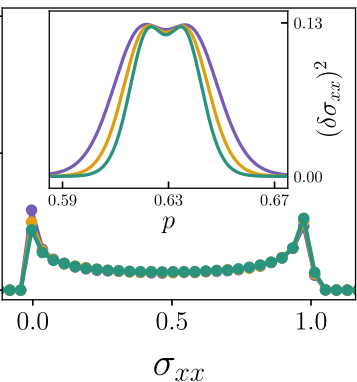

(c)

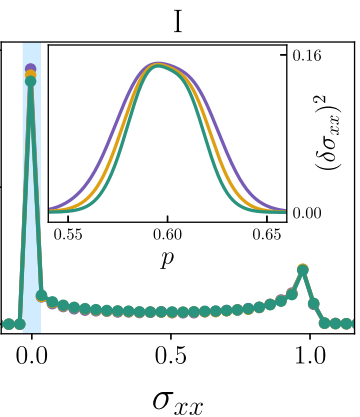

(d)

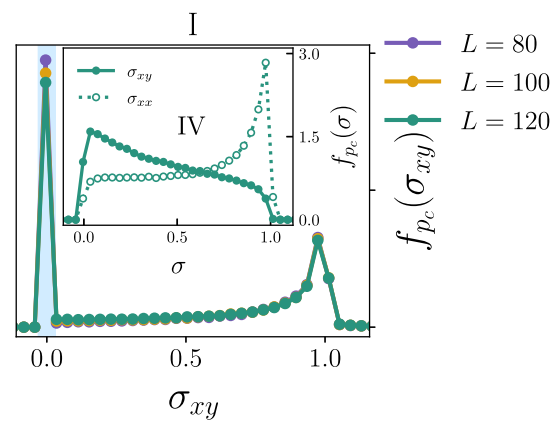

FIG. 3. Evolution of CDs for the discrete (top row) and the continuum model (bottom row) along the phase boundary. The insets in panels (a)-(c) illustrate the variance of the distribution near critical density. The inset in panel (d) highlights the difference of the $\sigma_{x x}$ and $\sigma_{x y}$ distributions at high density. Distributions are generated from up to $10^{5}$ configurations.

picture that the critical wave functions essentially reflect the geometry of the underlying lattice. As the density is increased, these signatures evolve smoothly to a different form and the CDs share qualitative features of $\mathrm{QH}$ systems.

\section{DISCUSSION}

The discovered features of ATC, while striking in the light of the literature accumulated during the last four decades, do not contradict the conclusions of the conventional scaling theory in disordered systems. Despite the superficial similarity, the essential features of the transition on random lattices with varying density are not captured by disordered models on regular geometries. Varying $p$ introduces a variable length scale $l \propto\left|p-p_{c}^{c l}\right|^{-4 / 3}$ in the system, where $p_{c}^{c l}$ is the percolation threshold of the lattice. When $p>p_{c}^{c l}$, this scale characterizes the linear size of randomly placed holes in the lattice. The geometry near $p=1$ is described by dense system with isolated vacancies, while in the limit $p \rightarrow p_{c}^{c l}$ the holes on a lattice diverge $l \rightarrow \infty$, leaving only a fractal critical cluster at $p_{c}^{c l}$. In the dense system the geometric correlations have very short range, while they diverge at $p_{c}^{c l}$. Since the nature of correlations in the disordered systems are known to affect the universality class of the transition [64,67], it is natural to consider the variable scale $l$ of the geometric fluctuations as the source of the nonuniversality. Interestingly, when some aspects of geometric fluctuations were recently implemented in disordered models, the critical exponents were observed to exhibit variation $[35,37,68]$. We speculate that the reason for that behavior reflects the nonuniversal scaling established in the present work.
The present work has fundamental ramification on the rapidly growing field of amorphous topological systems. The first experimental realizations of elemental and artificial amorphous topological systems have recently become accessible. Thus, it is plausible that the remarkable aspects of ATC can soon be probed in experiments. A comprehensive characterization of ATC can be carried out by probing systems at different densities or variable geometric fluctuations. This could be most naturally carried out in designer systems $[5,14]$ where density of lattice sites or geometry of the lattice can be easily controlled. The present work also opens many new lines of research. For example, what are the consequences of ATC on other symmetry classes and dimensions such as recently studied amorphous $\mathrm{Bi}_{2} \mathrm{Se}_{3}$ [6]? How do the statistical properties of wave functions reflect the ATC? How are the dynamical properties affected? What new features will quenched disorder add to ATC? These questions will be studied in the future.

\section{SUMMARY}

In this work we studied critical transport in Chern insulators with random geometry and discovered remarkable amorphous scaling behavior. In striking contrast to conventional expectations, the critical exponents and critical conductance distributions characterizing the transition are strongly nonuniversal. Our results indicate that, by varying density without affecting symmetries, amorphous topological phase transitions interpolate between a geometric percolation-type and Anderson localization-type transitions. The discovered nonuniversal scaling is a generic feature of amorphous topological matter, indicating striking departure from conventional topological systems. 
[1] M. G. Vergniory, L. Elcoro, C. Felser, N. Regnault, B. A. Bernevig, and Z. Wang, A complete catalog of highquality topological materials, Nature (London) 566, 480 (2019).

[2] T. Zhang, Y. Jiang, Z. Song, H. Huang, Y. He, Z. Fang, H. Weng, and C. Fang, Catalogue of topological electronic materials, Nature (London) 566, 475 (2019).

[3] F. Tang, H. C. Po, A. Vishwanath, and X. Wan, Comprehensive search for topological materials using symmetry indicators, Nature (London) 566, 486 (2019).

[4] X. Li, P. Li, V. D.-H. Hou, M. DC, C.-H. Nien, F. Xue, D. Yi, C. Bi, C.-M. Lee, S.-J. Lin, et al., Large and robust charge-to-spin conversion in sputtered Weyl semimetal WTex with structural disorder, arXiv:2001.04054.

[5] P. Zhou, G.-G. Liu, X. Ren, Y. Yang, H. Xue, L. Bi, L. Deng, Y. Chong, and B. Zhang, Photonic amorphous topological insulator, Light: Sci. Appl. 9, 133 (2020).

[6] P. Corbae, S. Ciocys, D. Varjas, S. Zeltmann, C. H. Stansbury, M. Molina-Ruiz, Z. Chen, L.-W. Wang, A. M. Minor, A. G. Grushin, et al., Evidence for topological surface states in amorphous $\mathrm{Bi}_{2} \mathrm{Se}_{3}$, arXiv: 1910.13412 .

[7] N. P. Mitchell, L. M. Nash, D. Hexner, A. M. Turner, and W. T. M. Irvine, Amorphous topological insulators constructed from random point sets, Nat. Phys. 14, 380 (2018).

[8] D. C. Mahendra, R. Grassi, J.-Y. Chen, M. Jamali, D. R. Hickey, D. Zhang, Z. Zhao, H. Li, P. Quarterman, Y. Lv, et al., Roomtemperature high spin-orbit torque due to quantum confinement in sputtered $\mathrm{Bi}_{x} \mathrm{Se}_{(1-x)}$ films, Nat. Mater. 17, 800 (2018).

[9] A. Agarwala and V. B. Shenoy, Topological Insulators in Amorphous Systems, Phys. Rev. Lett. 118, 236402 (2017).

[10] Y.-B. Yang, T. Qin, D.-L. Deng, L.-M. Duan, and Y. Xu, Topological Amorphous Metals, Phys. Rev. Lett. 123, 076401 (2019).

[11] M. Costa, G. R. Schleder, M. Buongiorno Nardelli, C. Lewenkopf, and A. Fazzio, Toward realistic amorphous topological insulators, Nano Lett. 19, 8941 (2019).

[12] P. Mukati, A. Agarwala, and S. Bhattacharjee, Topological and conventional phases of a three-dimensional electronic glass, Phys. Rev. B 101, 035142 (2020).

[13] A. Agarwala, V. Juričić, and B. Roy, Higher-order topological insulators in amorphous solids, Phys. Rev. Res. 2, 012067 (2020).

[14] K. Pöyhönen, I. Sahlberg, A. Westström, and T. Ojanen, Amorphous topological superconductivity in a Shiba glass, Nat. Commun. 9, 2103 (2018).

[15] M. Xiao and S. Fan, Photonic Chern insulator through homogenization of an array of particles, Phys. Rev. B 96, 100202(R) (2017).

[16] T. Mano and T. Ohtsuki, Application of convolutional neural network to quantum percolation in topological insulators, J. Phys. Soc. Jpn. 88, 123704 (2019).

[17] D. Varjas, M. Fruchart, A. R. Akhmerov, and P. M. PerezPiskunow, Computation of topological phase diagram of disordered $\mathrm{Pb}_{1-x} \mathrm{Sn}_{x} \mathrm{Te}$ using the kernel polynomial method, Phys. Rev. Res. 2, 013229 (2020).

[18] Q. Marsal, D. Varjas, and A. G. Grushin, Topological WeaireThorpe models of amorphous matter, Proc. Nat. Acad. Sci., 202007384 (2020).

[19] A. G. Grushin, Topological phases of amorphous matter, arXiv:2010.02851.
[20] B. Focassio, G. R. Schleder, M. Costa, A. Fazzio, and C. Lewenkopf, Structural and electronic properties of realistic two-dimensional amorphous topological insulators, arXiv:2010.14239.

[21] P. Corbae, F. Hellman, and S. M. Griffin, Structural disorderdriven topological phase transition in noncentrosymmetric BiTel, arXiv:2010.07456.

[22] D. E. Khmel'nitskii, Quantization of Hall conductivity, JETP Lett. 38, 552 (1983).

[23] A. M. M. Pruisken, Dilute instanton gas as the precursor to the integral quantum Hall effect, Phys. Rev. B 32, 2636 (1985).

[24] B. Huckestein, Scaling theory of the integer quantum Hall effect, Rev. Mod. Phys. 67, 357 (1995).

[25] F. Evers and A. D. Mirlin, Anderson transitions, Rev. Mod. Phys. 80, 1355 (2008)

[26] S. Koch, R. J. Haug, K. V. Klitzing, and K. Ploog, SizeDependent Analysis of the Metal-Insulator Transition in the Integral Quantum Hall Effect, Phys. Rev. Lett. 67, 883 (1991).

[27] L. W. Engel, D. Shahar, C. Kurdak, and D. C. Tsui, Microwave Frequency Dependence of Integer Quantum Hall Effect: Evidence for Finite-Frequency Scaling, Phys. Rev. Lett. 71, 2638 (1993).

[28] H. P. Wei, D. C. Tsui, M. A. Paalanen, and A. M. M. Pruisken, Experiments on Delocalization and University in the Integral Quantum Hall Effect, Phys. Rev. Lett. 61, 1294 (1988).

[29] W. Li, G. A. Csáthy, D. C. Tsui, L. N. Pfeiffer, and K. W. West, Scaling and Universality of Integer Quantum Hall Plateau-ToPlateau Transitions, Phys. Rev. Lett. 94, 206807 (2005).

[30] C.-Z. Chang, W. Zhao, J. Li, J. K. Jain, C. Liu, J. S. Moodera, and M. H. W. Chan, Observation of the Quantum Anomalous Hall Insulator to Anderson Insulator Quantum Phase Transition and its Scaling Behavior, Phys. Rev. Lett. 117, 126802 (2016).

[31] W. Li, C. L. Vicente, J. S. Xia, W. Pan, D. C. Tsui, L. N. Pfeiffer, and K. W. West, Scaling in Plateau-To-Plateau Transition: A Direct Connection of Quantum Hall Systems with the Anderson Localization Model, Phys. Rev. Lett. 102, 216801 (2009).

[32] M. Amado, A. V. Malyshev, A. Sedrakyan, and F. DomínguezAdame, Numerical Study of the Localization Length Critical Index in a Network Model of Plateau-Plateau Transitions in the Quantum Hall Effect, Phys. Rev. Lett. 107, 066402 (2011).

[33] M. Puschmann, P. Cain, M. Schreiber, and T. Vojta, Integer quantum Hall transition on a tight-binding lattice, Phys. Rev. B 99, 121301(R) (2019).

[34] Q. Zhu, P. Wu, R. N. Bhatt, and X. Wan, Localization-length exponent in two models of quantum Hall plateau transitions, Phys. Rev. B 99, 024205 (2019).

[35] A. Klümper, W. Nuding, and A. Sedrakyan, Random network models with variable disorder of geometry, Phys. Rev. B 100, 140201(R) (2019).

[36] W. Nuding, A. Klümper, and A. Sedrakyan, Localization length index and subleading corrections in a Chalker-Coddington model: A numerical study, Phys. Rev. B 91, 115107 (2015).

[37] I. A. Gruzberg, A. Kluemper, W. Nuding, and A. Sedrakyan, Geometrically disordered network models, quenched quantum gravity, and critical behavior at quantum Hall plateau transitions, Phys. Rev. B 95, 125414 (2017).

[38] H. Obuse, I. A. Gruzberg, and F. Evers, Finite-Size Effects and Irrelevant Corrections to Scaling Near the Integer Quantum Hall Transition, Phys. Rev. Lett. 109, 206804 (2012). 
[39] K. Slevin and T. Ohtsuki, Critical exponent for the quantum Hall transition, Phys. Rev. B 80, 041304(R) (2009).

[40] H. Obuse, A. R. Subramaniam, A. Furusaki, I. A. Gruzberg, and A. W. W. Ludwig, Conformal invariance, multifractality, and finite-size scaling at Anderson localization transitions in two dimensions, Phys. Rev. B 82, 035309 (2010).

[41] I. C. Fulga, F. Hassler, A. R. Akhmerov, and C. W. J. Beenakker, Topological quantum number and critical exponent from conductance fluctuations at the quantum Hall plateau transition, Phys. Rev. B 84, 245447 (2011).

[42] Y. Huo and R. N. Bhatt, Current Carrying States in the Lowest Landau Level, Phys. Rev. Lett. 68, 1375 (1992).

[43] M. Puschmann, P. Cain, M. Schreiber, and T. Vojta, Edge state critical behavior of the integer quantum Hall transition, arXiv:2004.01611.

[44] I. Sahlberg, A. Westström, K. Pöyhönen, and T. Ojanen, Topological phase transitions in glassy quantum matter, Phys. Rev. Res. 2, 013053 (2020).

[45] C. W. Groth, M. Wimmer, A. R. Akhmerov, and X. Waintal, Kwant: a software package for quantum transport, New J. Phys. 16, 063065 (2014).

[46] See Supplemental Materials at http://link.aps.org/supplemental/ 10.1103/PhysRevResearch.2.043301 for technical details and supplementary results.

[47] S. Datta, Electronic Transport in Mesoscopic Systems (Cambridge University Press, Cambridge, 1997).

[48] S.-Q. Shen, Topological Insulators: Dirac Equation in Condensed Matter (Springer, Berlin, 2018) .

[49] K. Slevin and T. Ohtsuki, Corrections to Scaling at the Anderson Transition, Phys. Rev. Lett. 82, 382 (1999).

[50] W. H. Press, S. A. Teukolsky, W. T. Vetterling, and B. P. Flannery, Numerical Recipes 3rd Edition: The Art of Scientific Computing (Cambridge University Press, Cambridge, 2007) .

[51] D. Stauffer and A. Aharony, Introduction to Percolation Theory (Taylor \& Francis, London, 1992) .

[52] X. Wang, Q. Li, and Costas M. Soukoulis, Scaling properties of conductance at integer quantum Hall plateau transitions, Phys. Rev. B 58, 3576 (1998).

[53] Z. Wang, Božidar Jovanović, and Dung-Hai Lee, Critical Conductance and its Fluctuations at Integer Hall Plateau Transitions, Phys. Rev. Lett. 77, 4426 (1996).

[54] Y. Xue and E. Prodan, Quantum criticality at the Chernto-normal insulator transition, Phys. Rev. B 87, 115141 (2013).
[55] Miklós Antal Werner, A. Brataas, F. Von Oppen, and G. Zaránd, Anderson localization and quantum Hall effect: Numerical observation of two-parameter scaling, Phys. Rev. B 91, 125418 (2015).

[56] L. Schweitzer and P. Markoš, Universal Conductance and Conductivity at Critical Points in Integer Quantum Hall Systems, Phys. Rev. Lett. 95, 256805 (2005).

[57] F. Evers, A. Mildenberger, and A. D. Mirlin, Multifractality at the Quantum Hall Transition: Beyond the Parabolic Paradigm, Phys. Rev. Lett. 101, 116803 (2008).

[58] H. Obuse, A. R. Subramaniam, A. Furusaki, I. A. Gruzberg, and A. W. W. Ludwig, Boundary Multifractality at the Integer Quantum Hall Plateau Transition: Implications for the Critical Theory, Phys. Rev. Lett. 101, 116802 (2008).

[59] S. Cho and M. P. A. Fisher, Conductance fluctuations at the integer quantum Hall plateau transition, Phys. Rev. B 55, 1637 (1997).

[60] A. G. Galstyan and M. E. Raikh, Localization and conductance fluctuations in the integer quantum Hall effect: Real-space renormalization-group approach, Phys. Rev. B 56, 1422 (1997).

[61] Daniel P. Arovas, M. Janssen, and B. Shapiro, Real-space renormalization of the Chalker-Coddington model, Phys. Rev. B 56, 4751 (1997).

[62] Božidar Jovanović and Z. Wang, Conductance Correlations Near Integer Quantum Hall Transitions, Phys. Rev. Lett. 81, 2767 (1998).

[63] K. Slevin, T. Ohtsuki, and T. Kawarabayashi, Topology Dependent Quantities at the Anderson Transition, Phys. Rev. Lett. 84, 3915 (2000).

[64] P. Cain, R. A. Römer, M. Schreiber, and M. E. Raikh, Integer quantum Hall transition in the presence of a long-rangecorrelated quenched disorder, Phys. Rev. B 64, 235326 (2001).

[65] B. Kramer, T. Ohtsuki, and S. Kettemann, Random network models and quantum phase transitions in two dimensions, Phys. Rep. 417, 211 (2005).

[66] Rudolf A. Römer, Percolation, Renormalization and Quantum Hall Transition, in Computational Statistical Physics (Springer, 2002,) pp. 279-294.

[67] N. Sandler, H. R. Maei, and J. Kondev, Correlated quantum percolation in the lowest Landau level, Phys. Rev. B 70, 045309 (2004).

[68] C.-Z. Chen, H. Liu, and X. C. Xie, Effects of Random Domains on the Zero Hall Plateau in the Quantum Anomalous Hall Effect, Phys. Rev. Lett. 122, 026601 (2019). 